\title{
Loss of membranous Ep-CAM in budding colorectal carcinoma cells
}

\author{
Marleen JEM Gosens ${ }^{1}$, Léon CL van Kempen ${ }^{1}$, Cornelis JH van de Velde ${ }^{2}$, \\ J Han JM van Krieken ${ }^{1}$ and Iris D Nagtegaal ${ }^{1}$ \\ ${ }^{1}$ Department of Pathology, Radboud University Nijmegen Medical Centre, Nijmegen, The Netherlands and \\ ${ }^{2}$ Department of Surgery, Leiden University Medical Centre, Leiden, The Netherlands
}

\begin{abstract}
Tumor budding is a histological feature that reflects loss of adhesion of tumor cells and is associated with locoregional metastasis of colorectal carcinoma. Although nuclear localization of $\beta$-catenin is associated with tumor budding, the molecular mechanism remains largely elusive. In this study, we hypothesize that the epithelial cell adhesion molecule (Ep-CAM) is involved in tumor budding. In order to address this question, we performed immunohistochemistry on Ep-CAM using three different antibodies (monoclonal antibodies Ber-ep4 and 311-1K1 and a polyclonal antibody) and a double staining on $\beta$-catenin and Ep-CAM. In addition, Ep-CAM mRNA was monitored with mRNA in situ hybridization. Subsequently, we determined the effect of Ep-CAM staining patterns on tumor spread in rectal cancer. In contrast to the tumor mass, budding cells of colorectal carcinoma displayed lack of membranous but highly increased cytoplasmic Ep-CAM staining and nuclear translocation of $\beta$-catenin. mRNA in situ hybridization suggested no differences in Ep-CAM expression between the invasive front and the tumor mass. Importantly, reduced Ep-CAM staining at the invasive margin of rectal tumor specimens $(n=133)$ correlated significantly with tumor budding, tumor grade and an increased risk of local recurrence $(P=0.001, P=0.04$ and $P=0.03$, respectively). These data demonstrate abnormal processing of Ep-CAM at the invasive margin of colorectal carcinomas. Our observations indicate that loss of membranous Ep-CAM is associated with nuclear $\beta$-catenin localization and suggest that this contributes to reduced cell-cell adhesions, increased migratory potential and tumor budding.
\end{abstract}

Modern Pathology (2007) 20, 221-232. doi:10.1038/modpathol.3800733

Keywords: Ep-CAM; adhesion; budding; colorectal cancer; tumor front; recurrence

Budding of dedifferentiated tumor cells at the invasive margin is well described in colorectal cancer. Tumor budding or sprouting is a histologic feature of loss of adhesion and is associated with locoregional tumor spread. ${ }^{1,2}$ These budding tumor cells have been described to exhibit nuclear $\beta$ catenin. ${ }^{3}$ In the nucleus, $\beta$-catenin can activate the transcription of genes associated with cell proliferation and differentiation. ${ }^{4}$ When $\beta$-catenin is bound to the cytoplasmic domain of E-cadherin, it enables E-cadherin to function as a cell-cell adhesion molecule and mediates the interplay of adherens junction molecules with the actin cytoskeleton. ${ }^{4}$ Aberrations of E-cadherin expression can lead to abundant cytoplasmic $\beta$-catenin and subsequent nuclear localization. Based on these well-described

Correspondence: Dr MJEM Gosens, MSc, Department of Pathology 437 PA, Radboud University Nijmegen Medical Centre, PO Box 9101, Nijmegen 6500 HB, The Netherlands.

E-mail: m.gosens@pathol.umcn.nl

Received 2 June 2006; revised 22 September 2006; accepted 6 November 2006 interactions between $\beta$-catenin and E-cadherin, it is plausible that modulation of E-cadherin-mediated adhesion is involved in tumor budding. However, Masaki et $a l^{5}$ did not find a correlation between nuclear $\beta$-catenin and changes of E-cadherin characteristics in budding colorectal tumor cells.

In addition to E-cadherin, the epithelial cell adhesion molecule (Ep-CAM, also known as BerEP4, CO17-1A, GA733 and EGP2) can also mediate cell-cell adhesion of epithelial cells. The adhesions between epithelial cells are facilitated by the extracellular domain of the protein. ${ }^{6}$ The smaller intracellular domain is known to interact with the actin skeleton via $\alpha$-actinin. ${ }^{7}$ Ep-CAM does not share significant homology with any of the four major families of adhesion molecules (cadherins, selectins, integrins and the immunoglobulins). Increased Ep-CAM expression is associated with enhanced proliferation and a lower differentiation grade of epithelial cells under nonpathological conditions. In the proliferative phase, Ep-CAM expression is associated with epithelial tissue remodeling. After cell proliferation, Ep-CAM expression declines and cellular differentiation initiates. An interesting 
example illustrating the correlations between EpCAM expression and cell proliferation in normal tissue is the hair follicle, where Ep-CAM is only expressed by cells in the highly proliferative zone. ${ }^{8}$ Furthermore, differential Ep-CAM expression is observed during embryonic development of different tissues such as pancreas and lung. ${ }^{9,10}$ In these perspectives, Ep-CAM can thus be regarded as a morphoregulatory protein.

Among neoplastic tissues, Ep-CAM is abundantly expressed in tumors of epithelial origin, for example, lung, breast, prostate, renal cell and colorectal carcinoma. ${ }^{11,12}$ Ep-CAM is present in high amounts in normal colorectal tissue, but its expression increases in polyps ${ }^{13}$ and carcinomas. ${ }^{14}$ In tumor cells, increased Ep-CAM expression promotes cellcell adhesion. Ectopic expression of the murine EpCAM orthologue (mEGP) by murine colorectal carcinoma cells increased cell-cell adhesion, attenuated tumor cell invasion in Matrigel, and decreased tumor incidence and metastasis when inoculated into the spleen of mice. ${ }^{15}$ Thus, these data suggest that Ep-CAM expression antagonizes tumor growth and metastasis. Because tumor budding is well described in colorectal cancer and is associated with poor prognosis, ${ }^{1,2}$ we hypothesized that altered Ep-CAM characteristics in rectal cancer may correlate with tumor budding and poor disease outcome. To this end, we have performed a detailed analysis of intratumoral Ep-CAM staining patterns using three different antibodies, and correlated these patterns with local and distant recurrence rates in patients with rectal cancer. By using two monoclonal antibodies with different epitopes on the Ep-CAM ectodomain, we could closely monitor changes in epitope availability of this domain. The combination of these two monoclonal antibodies with a polyclonal antibody, which recognizes the whole Ep-CAM protein (Figure 1), enabled us to study subcellular distributions of Ep-CAM and possible modifications of the protein such as cleavage or aberrant localization.

\section{Materials and methods}

\section{Immunohistochemistry}

Immunohistochemical staining of Ep-CAM was performed on paraffin-embedded tissue sections using monoclonal antibodies Ber-EP4 (1:100, Dako, Glostrup, Denmark) and 311-1k1 (1:50, Pickcell Laboratories, Leiden, The Netherlands; 1:50). The localization of their epitopes is depicted in Figure 1. In case of $\beta$-catenin, immunohistochemical staining was performed with the Clone 14 monoclonal antibody (1:6000, Transduction Laboratories, Lexington, USA). The ABC (avidin-biotin complex, Vector, Burlingame, USA) method was used for visualization with 3, 3'-diaminobenzide hydrochloride solution (DAB) or fast blue. Paraffin sections were de-waxed and rehydrated. All reactions were

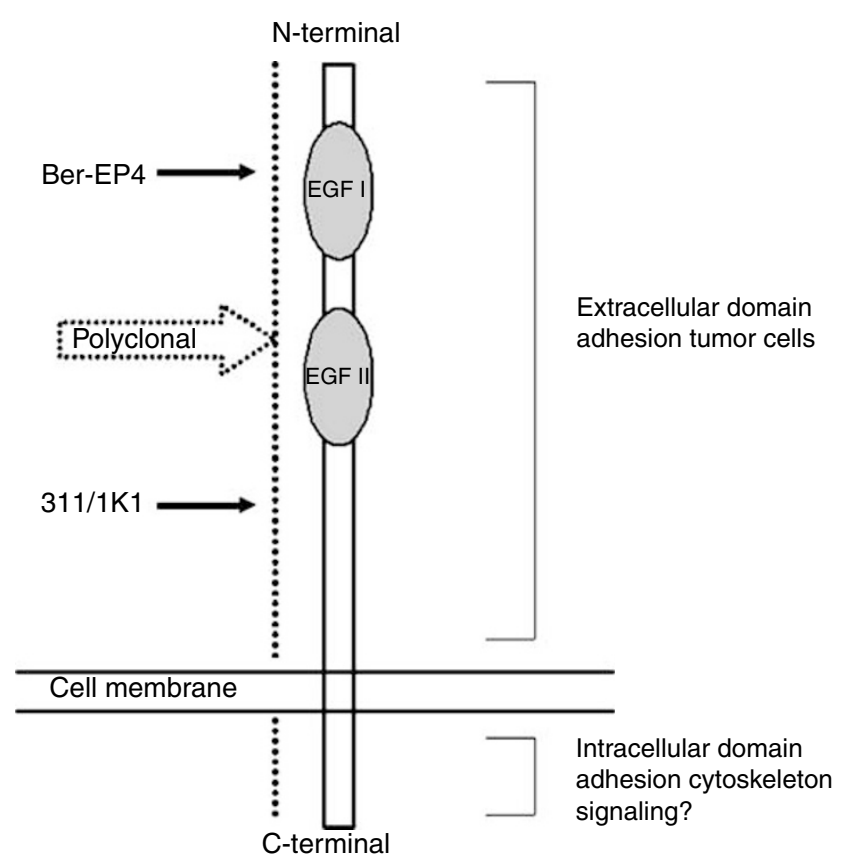

Figure 1 Schematic overview of the Ep-CAM protein with the positions of the Ber-EP4 and 311-1K1 epitopes and binding sites of the polyclonal antibody. Ber-EP4 and 311-1K1 are monoclonal antibodies that have specific epitopes (bold arrows). The polyclonal antibody recognizes numerous parts of the EP-CAM protein as depicted by the dotted line.

performed at room temperature, unless stated otherwise. Endogenous peroxidase activity was blocked by incubation in phosphate-buffered saline (PBS) containing $3 \% \mathrm{H}_{2} \mathrm{O}_{2}$ for $30 \mathrm{~min}$. After rinsing with PBS, antigen retrieval for Ber-EP4 staining consisted of incubation with $1 \%$ pronase at $37^{\circ} \mathrm{C}$ for $10 \mathrm{~min}$. Retrieval for $311-1 \mathrm{~K} 1$ and $\beta$-catenin staining involved microwave boiling in a $10 \mathrm{mM}$ sodium citrate buffer ( $\mathrm{pH} \mathrm{6.0)}$ for $10 \mathrm{~min}$. After boiling, the slides were allowed to cool down for at least $30 \mathrm{~min}$. After rinsing with PBS, slides were pretreated with $20 \%$ normal horse serum for $10 \mathrm{~min}$ to reduce nonspecific staining. All sera and antibodies were dissolved in PBS with 1\% BSA. Subsequently, slides were incubated in a humidity chamber with the primary antibody at $4^{\circ} \mathrm{C}$ for $16-20 \mathrm{~h}$. This long incubation time results in saturation of the reaction and therefore optimal staining. Staining of the DAB substrate was intensified with a $0.5 \%$ copper sulfate solution, in case of double staining this step was omitted. Slides were counterstained with hematoxylin solution or Nuclear Fast Red for 1 min, dehydrated and enclosed with Permount (Fisher Chemicals, NJ, USA).

A complete loss of Ber-EP4 staining was never observed and no distinction was made between the various degrees of decrease which were very subtle. Therefore, staining patterns of the Ber-EP4 antibody were categorized as follows: (i) no decrease of staining, (ii) decrease of staining at the front or (iii) decreased staining throughout the tumor. 


\section{Immunofluorescence}

Immunofluorescence double stainings were performed with Ber-EP4 and a chicken polyclonal anti-Ep-CAM antibody (kindly provided by Dr S Litvinov) and with Ber-EP4 and a monoclonal anticytokeratin antibody, Cam 5.2 (1:40, Becton Dickinson, Franklin Lakes, USA). Briefly after dewaxing and rehydration, slides were rinsed with PBS and incubated in this solution at $4{ }^{\circ} \mathrm{C}$ for at least $16 \mathrm{~h}$ to reduce auto-fluorescence. In case of Ber-EP4 and CAM 5.2, double staining antigen retrieval involved incubation with $1 \%$ pronase at $37^{\circ} \mathrm{C}$ for $10 \mathrm{~min}$ antigen retrieval for the Ber-EP4 and EpCAM polyclonal antibody double staining consisted of microwave boiling in a $10 \mathrm{mM}$ sodium citrate buffer ( $\mathrm{pH}$ 6.0) for $10 \mathrm{~min}$. After cooling down and rinsing with PBS, slides were incubated with $20 \%$ normal goat serum for $10 \mathrm{~min}$. Slides were incubated with the primary antibody Ber-EP 4 at $4^{\circ} \mathrm{C}$ overnight. After rinsing, slides were incubated with goat antimouse IgG-conjugated Alexa 488/594 (1:200, Invitrogen Molecular Probes, Eugene, USA) for $30 \mathrm{~min}$. After rinsing, slides were incubated with $20 \%$ goat serum or $100 \%$ normal goat serum to reduce nonspecific binding of the polyclonal antibody. Subsequently, slides were incubated with the second primary antibody of choice at $4{ }^{\circ} \mathrm{C}$ overnight. The polyclonal antibody was detected with goat anti-chicken IgG-conjugated Alexa 594 (1:200, Invitrogen Molecular Probes) for $30 \mathrm{~min}$. CAM 5.2 binding was visualized with goat anti-mouse IgG2aconjugated Alexa 488 (1:200, Invitrogen Molecular Probes) for $30 \mathrm{~min}$. After washing in PBS, slides were stained with 4'-6-diamidino-2-phenylindole (DAPI) for $30 \mathrm{~s}$ and sections were enclosed in fluorteck (Euro-Diagnostica, Arnhem, The Netherlands). For analysis, a fluorescent microscopy (Leica, Solms, Germany) was used. Images were captured, using a $\times 5, \times 10, \times 20$ or $\times 40$ objective and were prepared with Adobe Photoshop version 7.0.

\section{Ep-CAM mRNA In Situ Hybridization}

A 420 bp human cDNA fragment was generated by reverse transcriptase-polymerase chain reaction (RTPCR) according to standard procedures. Briefly, total RNA isolated from the $\mathrm{CaCo}_{2}$ colon carcinoma cell line was isolated with Trizol (Gibco BRL) according to the manufacturer's protocol and $0.5 \mu \mathrm{g}$ was reverse-transcribed at $42^{\circ} \mathrm{C}$ for $1 \mathrm{~h}$ using SuperScript II (Promega) and oligo dT16-18 primer. Ep-CAM cDNA was amplified using specific primers $\left(5^{\prime}-\right.$ CTGGCCGTAAACTGCTTTGT and 5'-CTTCCTCTA GTGTTGCGCAA) and were based on the reported sequence of human Ep-CAM (gi:10439469). Amplification consisted of preincubation at $95^{\circ} \mathrm{C}$ for $5 \mathrm{~min}$ before adding Taq polymerase and then 30 cycles at $95^{\circ} \mathrm{C}$ for $1 \mathrm{~min}, 55^{\circ} \mathrm{C}$ for $30 \mathrm{~s}$ and $72^{\circ} \mathrm{C}$ for $30 \mathrm{~s}$. A PCR product of the predicted size was cloned into the
pGEM-T easy vector (Promega, Madison, USA) and sequence was verified. The plasmid containing the partial cDNA of Ep-CAM was linearized by digestion with PstI or SphI and antisense and sense riboprobes were synthesized using T7 and SP6 RNA polymerase, respectively, and dioxygenin-labelled rUTP (Roche, Basel, Switzerland) according to standard procedures. mRNA in situ hybridization of paraffin-embedded tissue sections was performed as described previously. ${ }^{16}$

\section{Patient Selection}

In order to study the effect of Ep-CAM distribution in colorectal tumors on local and distant recurrence, we used data obtained from the radiotherapy (RT) + Total Mesorectal Excision (TME) trial. The TME trial was initiated in the Netherlands and included 1530 patients from January 1996 until December 1999. ${ }^{17,18}$ This prospectively randomized trial evaluated TME surgery with or without preoperative radiotherapy $(5 \times 5 \mathrm{~Gy})$. To be eligible, patients had to have histologically confirmed rectal adenocarcinoma, without evidence of distant metastasis. Patients with previous or coexisting cancer and those who had previously undergone large-bowel surgery, chemotherapy or radiotherapy were excluded. The TME trial was approved by the medical ethics committees of all participating hospitals and after informed consent had been obtained, selected patients were randomized and assigned to either radiotherapy (5 Gy on each of five days) followed by TME, or to TME alone. Radiotherapeutical, surgical and pathological procedures were standardized and subjected to strict quality control. ${ }^{19}$ Outcome measures included local and distant recurrences, confirmed by radiographic imaging and/or histological diagnosis. As local recurrence rates are very low after TME surgery, an artificial selection of 160 patients was made to study the role of biological markers in both local and distant recurrence. ${ }^{20}$ Forty stage II and 40 stage III patients without local recurrence or distant metastasis, 40 with distant metastasis and without local recurrence and 40 patients with local recurrence and without distant metastasis were selected. Former results obtained by studying this population $^{20}$ indicated sufficient statistical power in this population. The selection implies that local and distant recurrence percentages cannot be extrapolated towards the total population studied. Patients were selected from both randomization arms to exclude possible therapy-related effects. From these 160 patients, tumor samples were available for EpCAM immunohistochemistry of 133 patients. The median follow-up was 41.7 months.

\section{Pathology Procedures}

Tumor staging was performed by the use of the tumor-node-metastasis (TNM) classification. ${ }^{21}$ Growth 
pattern assessment was performed according to Jass. A circumferential margin of $1 \mathrm{~mm}$ or less was considered positive. Histologic differentiation grade was classified as undifferentiated, poorly differentiated, moderately and well. The grading decision was based on the least differentiated area. ${ }^{22}$ Tumor budding at the invasive margin was assessed as described by Ueno et al. ${ }^{23,24}$ In case of 117 tumors (88\%), a representative HE staining of the tumor area assessed with Ber-EP4 could be obtained. After choosing a field where budding was most intensive, the number of budding foci was counted using a $\times 25$ microscope objective. A budding focus was defined as a single isolated tumor cell or a cluster of tumor cells composed of fewer than five cells. Subsequently, these data were categorized as follows: I: 0-4 budding foci, II: 5-9 budding foci, III: 10-19 budding foci and IV: more than 20 budding foci.

\section{Statistical Analysis}

Ep-CAM staining patterns of patients included in the Dutch TME trial were correlated with local, distant and overall recurrence using Kaplan-Meier curves and log-rank testing. Associations between Ep-CAM patterns and histopathological parameters were analyzed by $\chi^{2}$ testing. $P$-values of $<0.05$ (twotailed) were considered as statistically significant.

\section{Results}

\section{Loss of the Ber-EP4 Epitope in Budding Tumor Cells}

Normal colon mucosa was intensely stained with Ber-EP4 and 311-1K1 monoclonal antibodies (Figure 2a and b). In colorectal tumors, decreased staining was predominately observed in budding tumor cells at the invasive margin. Additionally, we found focally decreased staining with these antibodies within the tumor mass (Figure 2c-f). In order to identify potential Ber-EP4-negative tumor cells, we used an immunofluorescence (IF) double staining with Ber-EP4 and the tumor marker CAM 5.2 (cytokeratin 7 and 8). With this method, a pattern of decreased Ber-EP4 staining on isolated infiltrating tumor cells was observed (Figure 3a-c). To demonstrate a true correlation between localization of $\beta$ catenin and the presence or absence of the Ber-EP4 epitope, we performed double stainings. Double staining of Ber-EP4 and $\beta$-catenin showed that tumor cells with decreased Ber-EP4 immunoreactivity frequently displayed nuclear translocation of $\beta$ catenin (Figure 4a and b).

These data suggested either an inhibition of Ep-CAM mRNA expression or abnormal processing of Ep-CAM at the invasive front. Ep-CAM mRNA in situ hybridization and Ber-EP4 immunohistochemistry on consecutive sections demonstrated that mRNA expression in cells displaying decreased
Ber-EP4 staining was not reduced (Figure 5). Therefore, these results suggested that the focal loss of Ber-EP4 and 311-1K1 immunoreactivity was probably caused by abnormal processing of Ep-CAM.

\section{Cytoplasmic Patterns of Ep-CAM Immunoreactivity in Budding Tumor Cells}

We subsequently addressed whether loss of membranous immunoreactivity could also be detected with a polyclonal anti-Ep-CAM antibody. Double stainings with Ber-EP4 and the polyclonal antibody showed that a decreased Ber-EP4 staining was accompanied by cytoplasmic staining of Ep-CAM as visualized by the polyclonal antibody (Figure $6 \mathrm{~d}$ and f). This was predominantly observed in budding tumor cells or clusters. In normal mucosa, both antibodies showed a membranous staining pattern of Ep-CAM with equal staining intensity (Figure 6ac). These data demonstrated that loss of membranous Ep-CAM immunoreactivity coincided with increased cytoplasmic staining.

\section{Clinical Relevance of Heterogeneous Staining Patterns}

In order to analyze the prognostic value of decreased Ber-EP4 immunoreactivity in budding tumor cells, the relation between Ber-EP4 staining patterns and local, distant and overall recurrence was assessed by using data from the TME trial in which patients were randomized for TME surgery-only or radiotherapy followed by TME surgery. No correlations were found between the surgery-only and the irradiated group with respect to the immunohistochemical staining patterns. Furthermore, no correlations were found between Ep-CAM staining patterns and lymph node involvement, tumor depth, circumferential margin involvement and tumor stage (Table 1). Differentiation grade and presence or absence of membranous Ep-CAM at the invasive margin were found to be significantly correlated (Table 1). Tumors with selective loss of membranous EpCAM at the invasive margin were scored as poorly differentiated or undifferentiated in $35 \%$ of the cases in contrast to $20 \%$ of tumors with no loss of membranous Ep-CAM $(P=0.04)$. Differentiation grading alone failed to show significant correlations to local, distant or overall recurrence (data not shown), indicating that loss of membranous EpCAM expression in budding cells of rectal carcinoma is of higher prognostic value than tumor differentiation grade. In addition, a significant correlation was found between loss of membranous Ep-CAM and the extent of tumor budding (Table 1, $P=0.001$ ). Loss of membranous Ep-CAM was associated with a higher extent of tumor budding. This correlation between tumor budding and loss of membranous Ep-CAM is emphasized by the finding that tumor budding was also significantly correlated with tumor grade. A higher degree of budding was 

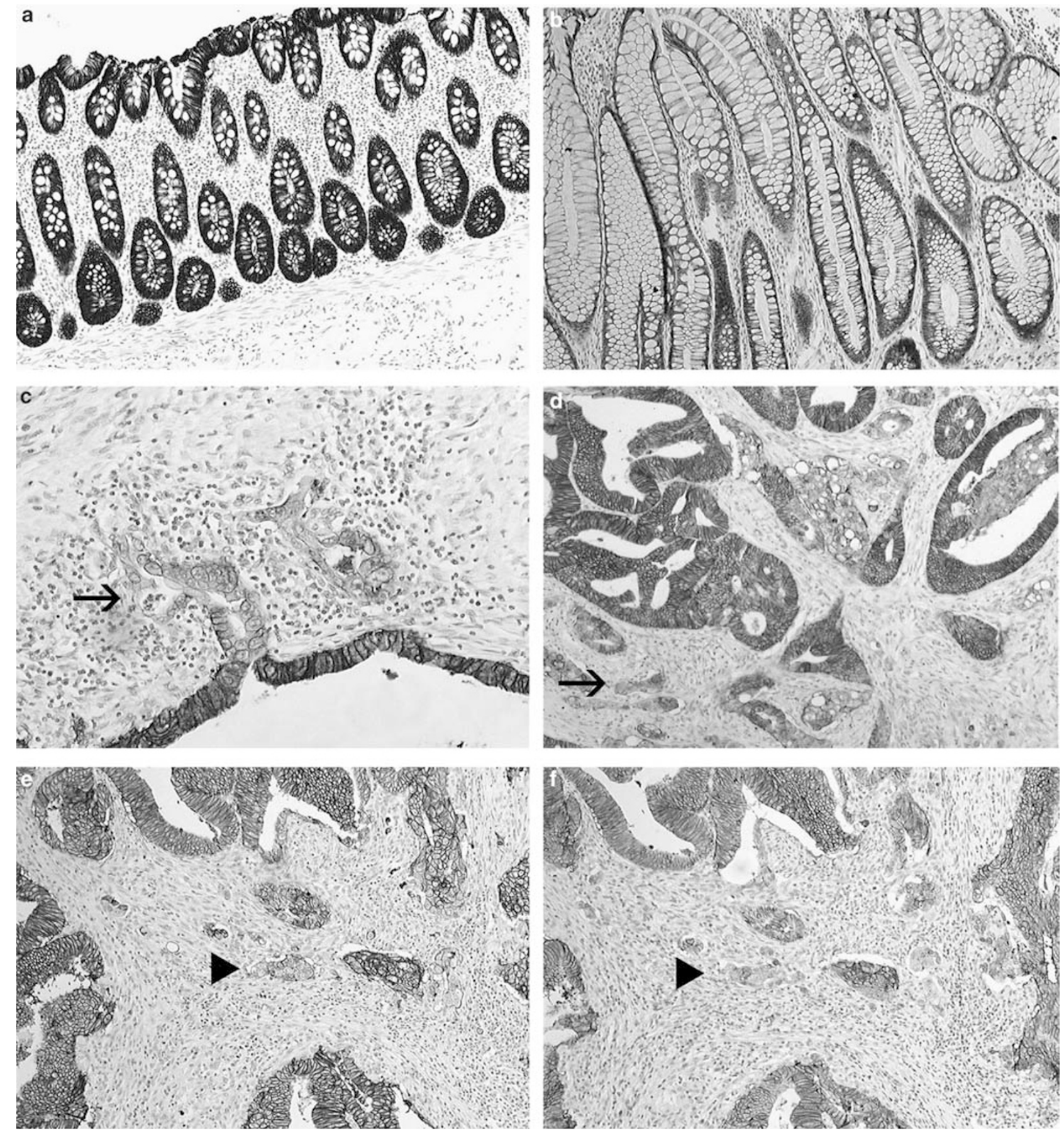

Figure 2 Representative Ep-CAM staining patterns in normal mucosa of the colon and colorectal adenocarcinoma with monoclonal antibodies Ber-EP4 and 311-1K1. (a) Normal mucosa stained with Ber-EP4 (original magnification: $\times 50$ ). (b) Normal mucosa stained with 311-1K1 (original magnification: $\times 100)$. (c) Decreased staining with Ber-EP4 in sprouting tumor cells, arrow (original magnification: $\times 200$ ). (d) Decreased staining of tumor mass with the 311-1K1 antibody, arrow (original magnification: $\times 50)$. (e and f) Serial sections with clusters of tumor cells stained with Ber-EP4 (e) and 311-1K1 (f) (original magnification: $\times 50$ ). Loss of immunoreactivity of isolated tumor cells and small clusters was observed with both 311-1K1 and with Ber-EP4 antibodies (arrowheads).

correlated with a lower degree of differentiation $\left(P=0.048, \chi^{2}\right.$ testing).

Tumors $(n=133)$ were stained and scored as (i) no decrease of Ep-CAM staining (70/133, 53\%), (ii) decrease of staining at the tumor front $(39 / 133,29 \%)$ or (iii) decrease of staining throughout the tumor (24/133, 18\%). Tumors with a decreased Ber-EP4 staining throughout the whole tumor were found to be a distinct group. This subpopulation of tumors behaved differently with respect to distant 

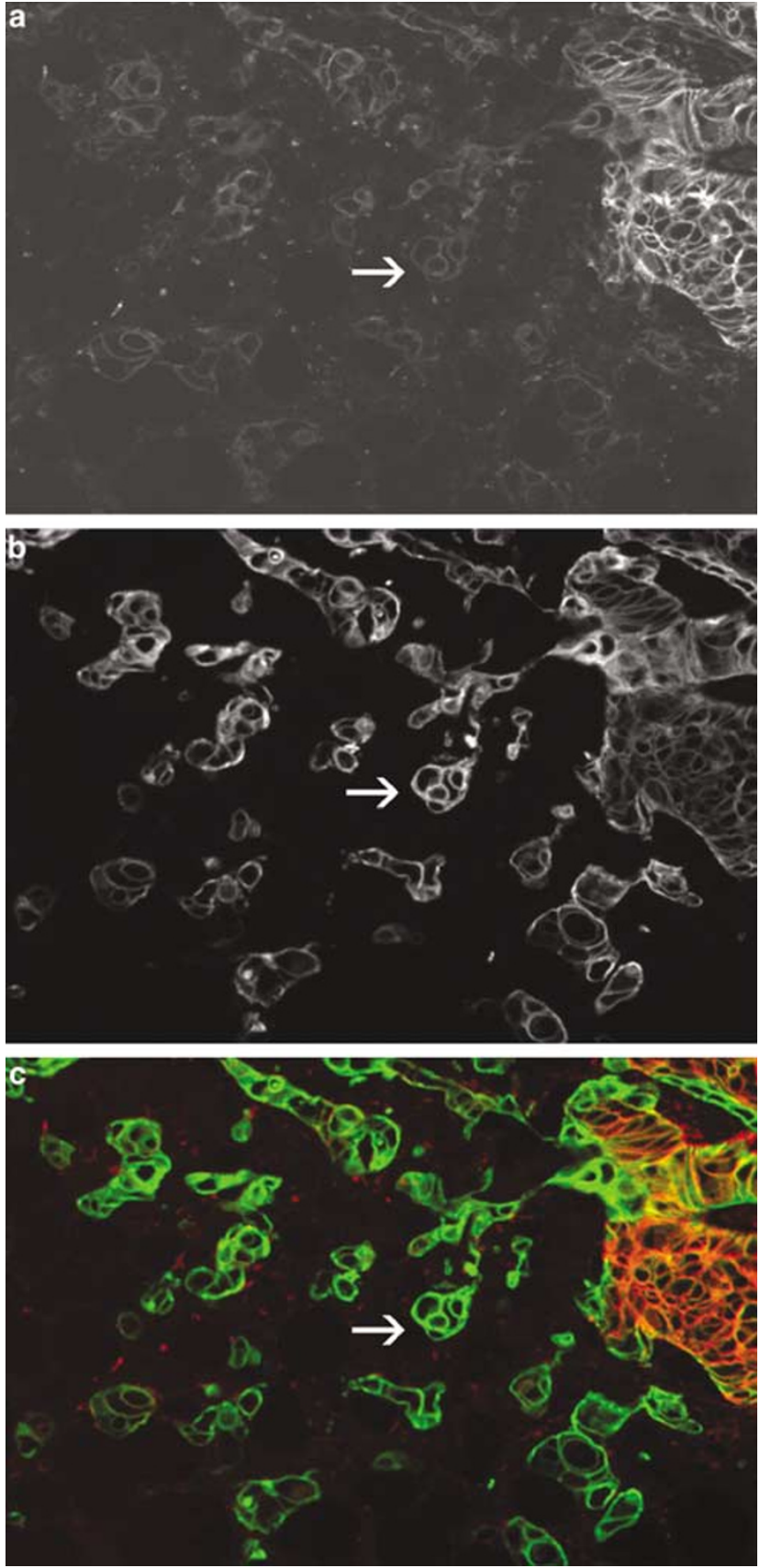

Figure 3 Immunofluoresence double staining of the invasive margin. (a) Ber-EP4 immunoreactivity. (b) Staining with the tumor marker cytokeratin 8. (c) Merge, Ber-EP4 staining is depicted in red, cytokeratin in green. Arrows indicate that infiltrating tumor cells show a loss of Ber-EP4 immunoreactivity (original magnifications: $\times 100$ ).

recurrence from tumors with decreased Ber-EP4 staining restricted to the tumor front. A loss of membranous Ep-CAM throughout the tumor was found to be correlated with an elevated risk on local recurrence alone compared to patients with no decrease of membranous Ep-CAM $(P=0.05)$. Moreover, in our opinion, this subpopulation is not suitable for studying the process of tumor budding

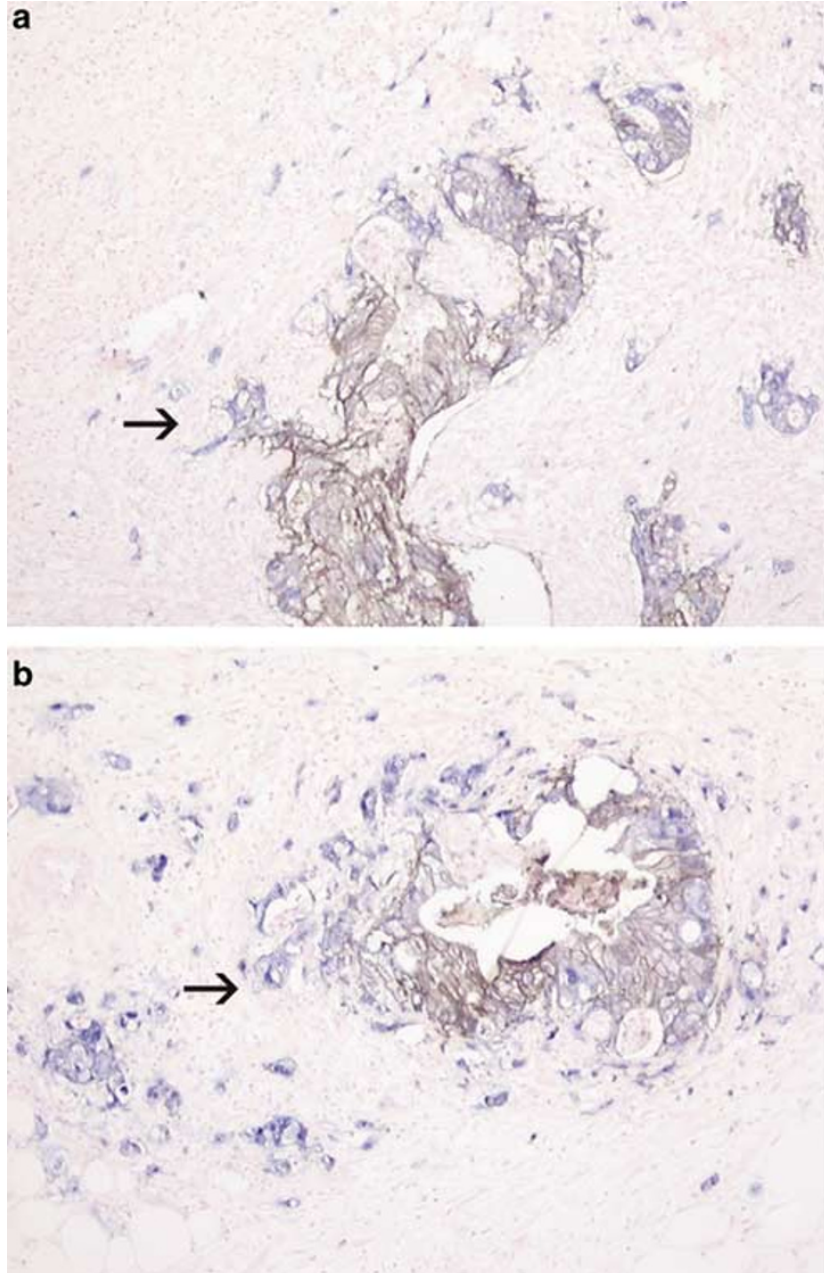

Figure 4 Double staining of Ep-CAM (Ber-EP4 epitope) and $\beta$-catenin of tumor masses with sprouting tumor cells. The Ber-EP4 epitope was visualized with DAB (brown) and $\beta$-catenin was displayed with fast blue staining (blue); nuclei were counterstained with nuclear fast red. (a) Tumor nodule with distinct budding of tumor cells that show a nuclear staining pattern of $\beta$-catenin and decreased staining of the Ber-EP4 epitope, arrows (original magnification: $\times 100$ ). This staining pattern of $\beta$-catenin was not observed centrally in the tumor nodule. (b) Budding tumor cells spreading into the mesorectal fat. Isolated tumor cells exhibit loss of the Ber-EP4 epitope and nuclear $\beta$-catenin as represented by an arrow (original magnification: $\times 100$ ).

at the invasive margin. Because this subpopulation has prognostic implications which are distinct from tumors with decreased Ber-EP4 staining exclusively observed at the front, it could interfere with the prognostic implications of the latter group. Because of this reason, the effect of the Ep-CAM characteristics at the tumor front was conducted in 109 (39+ 70) cases. Decreased staining intensity of Ber-EP4 at the invasive front was always accompanied by a focally infiltrating growth pattern. Loss of membranous staining at the infiltrative margin correlated with a significantly higher risk of local recurrence $(P=0.03$, Figure 7) compared with tumors with homogenous Ep-CAM patterns. A decrease of 

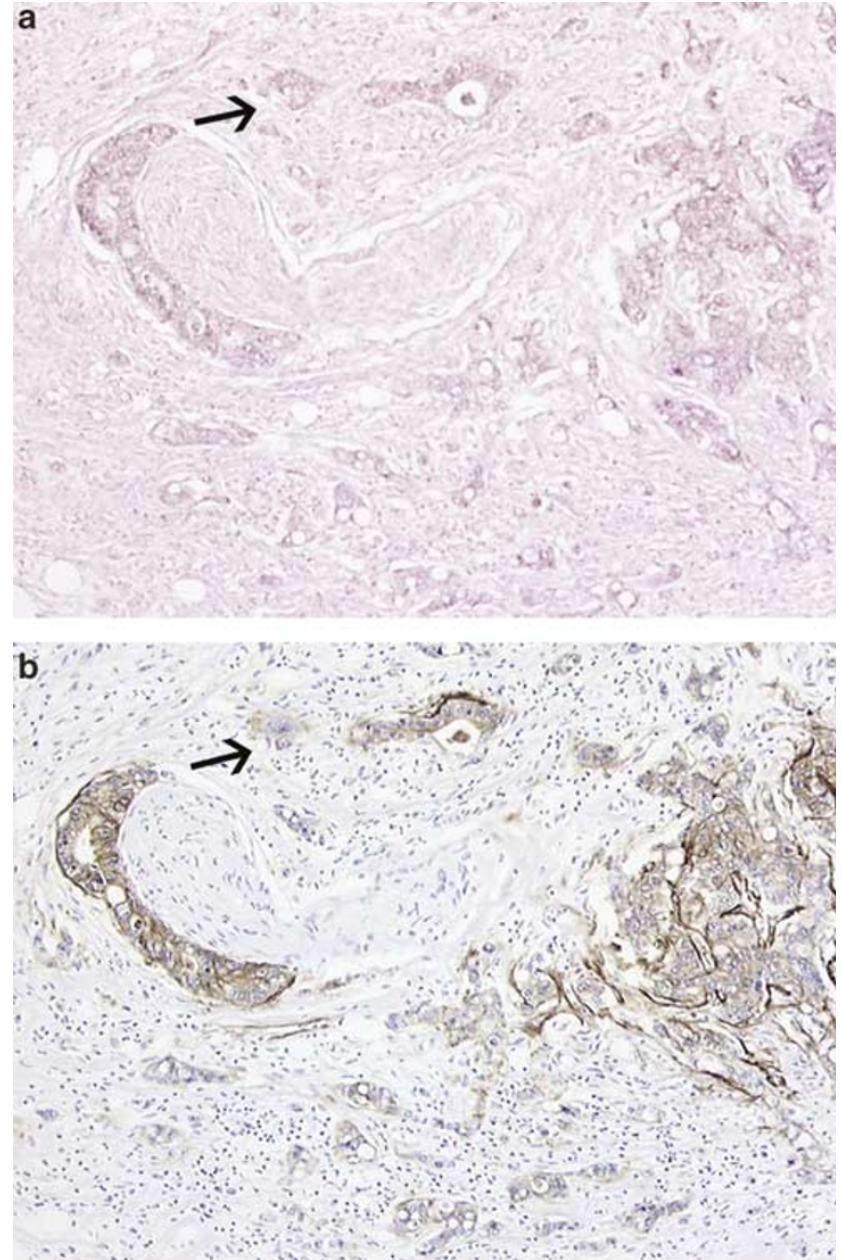

Figure 5 Ep-CAM mRNA in situ hybridization and Ber-EP4 immunohistochemistry on serial sections. (b) Ber-EP4 staining of clusters of tumors cells at the tumor front. (a) Ep-CAM mRNA ISH of the same tumor area. Tumor cells with decreased Ber-EP4 staining do not appear to have lower amounts of Ep-CAM mRNA (arrows, original magnification: $\times 100$ ).

Ep-CAM staining at the front indicated an elevated risk on distant recurrence, but this finding was not significant $(P=0.06)$. For overall recurrence, loss of membranous Ep-CAM strongly correlated with a significant higher risk of this event $(P=0.01)$.

\section{Discussion}

In this study, we demonstrate a focal loss of membranous Ep-CAM immunoreactivity at the invasive margin predominantly in sprouting tumor cells, which is frequently accompanied by nuclear $\beta$ catenin translocation. These findings strongly suggest that the morphoregulator Ep-CAM is involved in budding of rectal carcinoma.

Analysis of Ep-CAM mRNA expression in rectal carcinoma did not reveal strong differences in levels of transcription indicating that Ep-CAM at the invasive margin evidences abnormal post-transla- tional processing. It has to be emphasized that budding cells are not deprived from Ep-CAM. Posttranslational modifications did not affect Ep-CAM expression, that is, the polyclonal antibody showed that it was still present, but altered its cellular localization. Therefore, infiltrating cells possibly retain the features that contribute to high levels of Ep-CAM, for example, enhanced proliferation and loss of differentiation. ${ }^{25}$ Additionally, lack of extracellular Ep-CAM can enhance the migratory capacity by attenuating cell-cell adhesion.

The aberrant localization of Ep-CAM could contribute to tumor budding. During embryonic development of the pancreas, the highest levels of EpCAM expression are found in islet-like cell clusters budding from the ductal tree, suggesting that increased expression contributes to budding under nonpathological conditions. ${ }^{9}$ In contrast, we have found post-translational modification of Ep-CAM in budding tumor cells of rectal cancers in which EpCAM is already abundantly expressed. Assessment of the extent of tumor budding showed that loss of membranous Ep-CAM was significantly correlated to a higher extend of tumor budding. Furthermore, tumors with loss of membranous Ep-CAM at the invasive margin are more often graded as poorly or undifferentiated. Also, the clinical implications of tumor budding, that is, increased locoregional spread are reflected by the loss of membranous EpCAM staining as it correlated with increased local recurrence following surgical removal of the tumor. Tumor cells at the invasive front that exhibit loss of extracellular Ep-CAM have increased migratory potential and can spread through the bowel wall more easily. This is also reflected by the percentage of involved margins; 31 and $23 \%$ for tumors with and without loss of extracellular Ep-CAM at the tumor front respectively (Table 1). Increased local tumor spread owing to loss of extracellular Ep-CAM could account for noncurative resections and therefore increased local recurrence.

Loss of membranous Ep-CAM (Table 1) and tumor budding do not correlate with the TNM criteria. Although we do not have a proven explanation, it might be that tumor budding is a biological phenomenon that can occur at various moments during tumor development and is described to occur at various $\mathrm{T}$ stages including $\mathrm{T} 1$ and $\mathrm{T} 2{ }^{26,27}$ Thus, tumor budding reflects interaction at the level of the tumor microenvironment and is independent of the $\mathrm{T}$ stage itself. A significant correlation between loss of membranous Ep-CAM and lymph node (Table 1) and distant metastasis was also not observed (Figure 7). Invasion owing to loss of adhesion, although related to metastasis, is a distinct feature during tumor progression. In order to metastasize, tumor cells require more characteristics than increased invasive potential such as extracellular matrix remodeling, induction of angiogenesis and modulation of cell-cell and cell-matrix adhesive properties. 

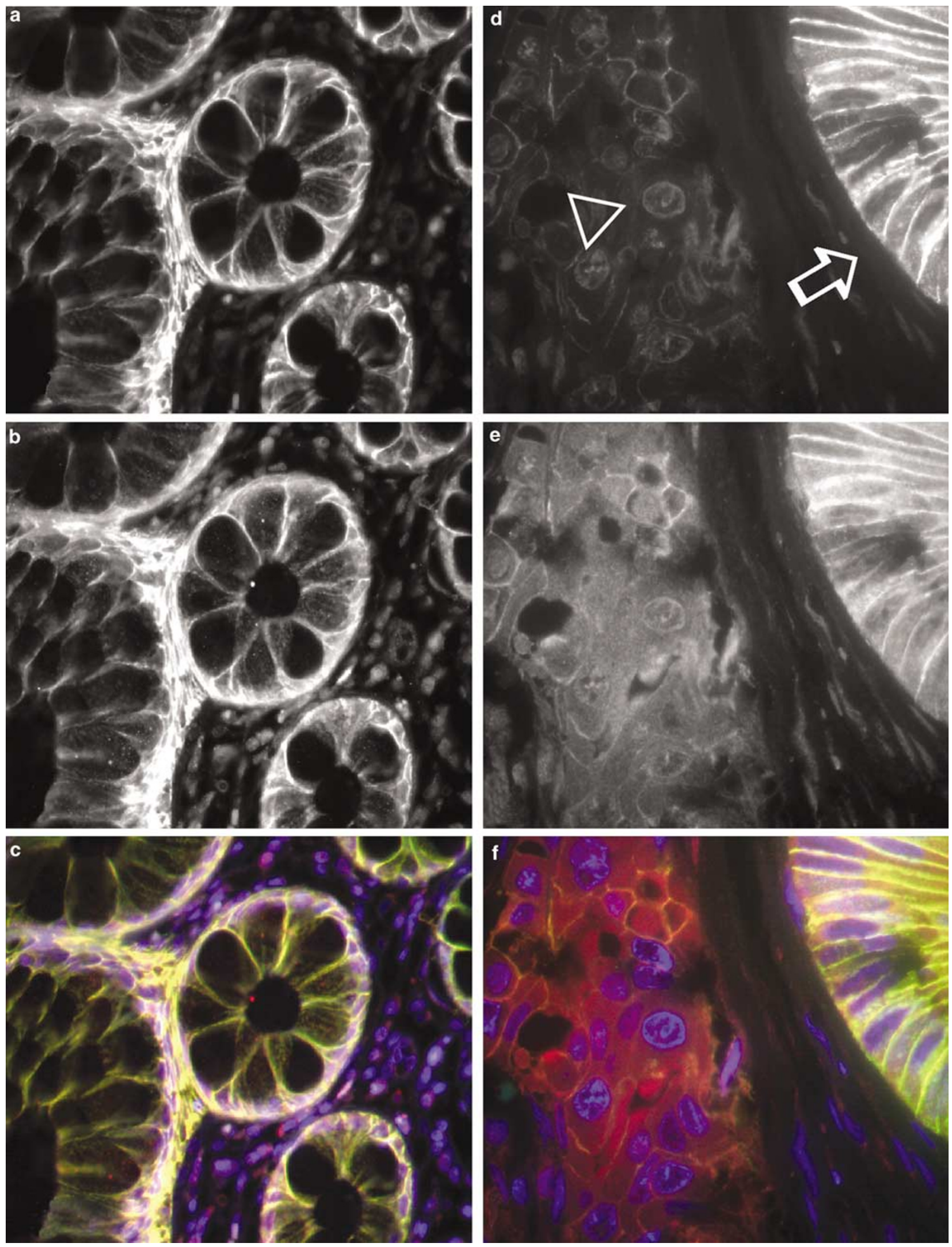
Infiltrating, sprouting tumor cells are discriminative from the tumor mass with respect to modifications of the Ep-CAM protein and thus suggest a role for this protein in the formation of tumor budding. Therefore, cytoplasmic Ep-CAM localization could alter its morphoregulatory capacity as compared to its membranous localization, and that sustains tumor budding.

Loss of membranous Ep-CAM can modulate changes of the cytoskeleton and hence change cell morphology. The extracellular domain of Ep-CAM with its two EGF-like repeats in a cystine-rich domain followed by a cystine-poor domain is similar to the organization of the extracellular domains of the lin21/Notch family. These proteins are involved in intercellular signaling and cell-cell interactions that are important for differentiation and segregation. ${ }^{28}$ It has been demonstrated that the cytoplasmic domain of Ep-CAM interacts with the actin cytoskeleton. ${ }^{7}$ Recently, it was demonstrated that high cytoplasmic expression of actinin-4, a modulator of the cytoskeleton, is predominantly observed in budding cells of colorectal carcinoma. ${ }^{29}$ Furthermore, high levels of actinin-4 are associated with increased cell motility. ${ }^{30}$ Although it remains elusive if there is a causal relation between cytoplasmic Ep-CAM and actinin-4 expression, it is tempting to speculate that both proteins are closely involved in tumor budding.

It remains elusive how Ep-CAM retains its cytoplasmic localization in budding tumor cells. The observed decrease in immunoreactivity of two monoclonal antibodies binding to the extracellular epitopes and a cytoplasmic staining pattern with the polyclonal antibody suggests internalization of Ep-CAM remnants after proteolytic modifications. Internalization of Ep-CAM has been reported previously by Litvinov et $a l^{31}$. Our finding that loss of

Table 1 Ep-CAM patterns in relation to clinicopathological factors

\begin{tabular}{|c|c|c|c|c|}
\hline \multirow[t]{2}{*}{ Factor } & \multirow[t]{2}{*}{ Category } & \multicolumn{2}{|c|}{ Ep-CAM pattern } & \multirow[t]{2}{*}{$\mathrm{P}$-value } \\
\hline & & Decrease at tumor front & No decrease at tumor front & \\
\hline \multirow[t]{2}{*}{ Randomization } & RT and surgery & $14(36 \%)$ & $30(43 \%)$ & \multirow[t]{2}{*}{0.31} \\
\hline & Surgery alone & $25(64 \%)$ & $40(57 \%)$ & \\
\hline \multirow[t]{2}{*}{ Lymph node involvement } & Positive lymph nodes & $27(69 \%)$ & $39(56 \%)$ & \multirow[t]{2}{*}{0.12} \\
\hline & No positive lymph nodes & $12(31 \%)$ & $31(44 \%)$ & \\
\hline \multirow[t]{3}{*}{ Tumor depth } & $\mathrm{T} 2$ & $9(23 \%)$ & $19(27 \%)$ & \multirow[t]{3}{*}{0.66} \\
\hline & T3 & $30(77 \%)$ & $50(72 \%)$ & \\
\hline & $\mathrm{T} 4$ & $0(0 \%)$ & $1(2 \%)$ & \\
\hline \multirow[t]{2}{*}{ Circumferential margin } & Negative & $27(69 \%)$ & $54(77 \%)$ & \multirow[t]{2}{*}{0.25} \\
\hline & Positive & $12(31 \%)$ & $16(23 \%)$ & \\
\hline \multirow[t]{2}{*}{ TNM stage } & Stage I/II & $11(28 \%)$ & $31(44 \%)$ & \multirow[t]{2}{*}{0.073} \\
\hline & Stage III/IV & $28(72 \%)$ & $39(56 \%)$ & \\
\hline \multirow[t]{2}{*}{ Differentiation grade } & Well/moderate & $24(62 \%)$ & $56(80 \%)$ & \multirow[t]{2}{*}{0.04} \\
\hline & Poor/undifferentiated & $15(38 \%)$ & $14(20 \%)$ & \\
\hline \multirow[t]{3}{*}{ Tumor budding } & I & $13(38 \%)$ & $43(69 \%)$ & \multirow[t]{3}{*}{0.001} \\
\hline & II & $14(41 \%)$ & $18(29 \%)$ & \\
\hline & III & $7(21 \%)$ & $1(2 \%)$ & \\
\hline Mean value smallest CRM (cm) & & 0.45 & 0.9 & $0.055^{*}$ \\
\hline
\end{tabular}

Abbreviation: RT, radiotherapy.

${ }^{*} P$-value calculated with Mann-Whitney test.

Bold values indicate statistical significance.

Figure 6 Immunofluorescence double staining of Ep-CAM with both monoclonal Ber- EP4 and polyclonal antibody. (a) Normal mucosa of the colon shows a membranous staining pattern with Ber-EP4. (b) The polyclonal antibody also presents a membranous staining pattern in normal colon mucosa. (c) Merge Ber-EP4 is visualized in green and the polyclonal antibodies in red, nuclei are counterstained with DAPI (blue). (d) An isolated tumor cluster (arrowhead) next to a tumor gland (arrow), immunohistochemistry was performed with the Ber-EP4 antibody. (e) Immunohistochemical expression of Ep-CAM evaluated with the polyclonal antibody. (f) Merge Ber-EP4 is visualized in green and the polyclonal antibody in red, nuclei are counterstained with DAPI (blue). Loss of Ber-EP4 staining intensity is associated with a cytoplasmic staining pattern with the polyclonal anti-Ep-CAM antibody (original magnifications: $\times 400$ ). 


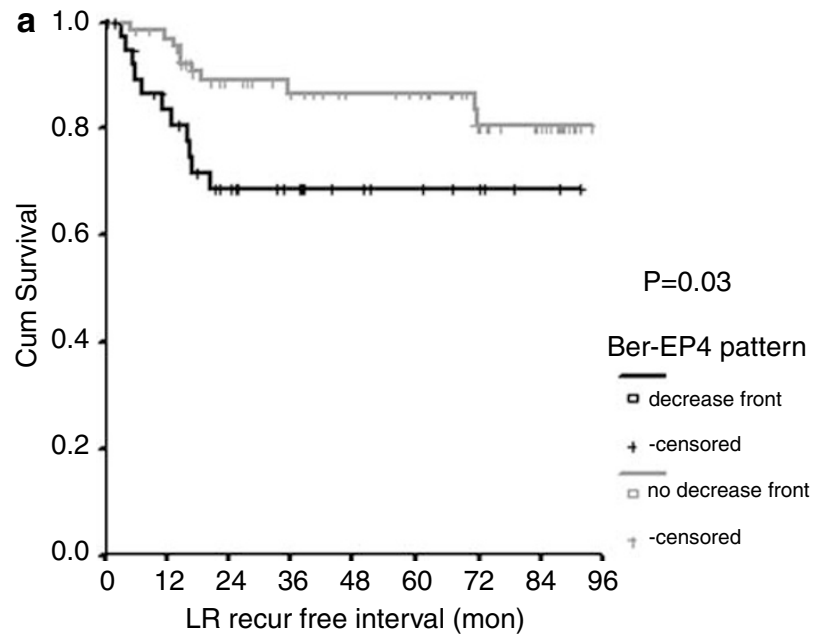

sible. The Lef/Tcf transcription factors, which are activated after $\beta$-catenin translocation, have been described to modulate the expression of different matrix metalloproteinases (MMP's) such as MMP-1 and MMP-7. ${ }^{32-34}$ As proteolysis of cell-cell adhesion molecules is a common feature in cancer, ${ }^{35}$ cleavage of Ep-CAM could be a downstream effect of nuclear translocation of $\beta$-catenin. This view is supported by a recent study by Hörkkö et $a{ }^{36}$ who postulate that nuclear accumulation of $\beta$-catenin is a requirement for tumor budding but other factors, which are more related to sprouting, also play role.

Alternatively, loss of membranous Ep-CAM in budding cells could be explained by splice variants of Ep-CAM. However, Balzar et al ${ }^{25}$ conducted an extensive analysis of Ep-CAM mRNAs in a large number of carcinoma cell lines and did not reveal any variations in Ep-CAM mRNA splicing. Furthermore, alternative glycosylation of the Ep-CAM ectodomain can shield Ber-EP4 and 311-1K1 epitopes. This in combination with impaired translocation to the membrane could also explain our findings. We have not addressed this possibility in our study.

Because high amounts of Ep-CAM are present on the membranes of many tumor types, it is an attractive target for immunotherapy. Since the 1990s, a number of trials investigated the therapeutic value of postoperative treatment with the 17-1A Ep-CAM antibody (Edrecolomab). ${ }^{37-39}$ This antibody also binds to EGF domain $\mathrm{I}^{40}$ and its epitope is in close proximity to Ber-EP4 (Figure 1). The results of these studies are conflicting, attributing positive, no or adverse effects of Edrecolomab therapy on survival and disease-free survival of patients with colorectal cancer. The findings presented in this study may explain these conflicting results. A subpopulation of tumor cells that were located at the invasive margin is undetectable for the Edrecolomab antibody. These cells lacked large amounts of extracellular Ep-CAM on their surfaces, which not only conceals them from the antibody, but also increased their migration capacity owing to attenuated Ep-CAM-mediated cell-cell adhesion.

\section{Acknowledgement}

We thank Dr SV Litvinov (PickCell Laboratories BV, Leiden, The Netherlands) for kindly providing the chicken polyclonal antibody towards Ep-CAM. Grants: Financial support was given by the: 'maag lever darm stichting', project no. WS 00-51.

Figure 7 Kaplan-Meier curves presenting the correlation between different Ep-CAM staining patterns and local recurrence (a), distant recurrence (b) and overall recurrence (c) $(N=109)$. Black line: selective decrease of Ber-EP4 staining at the invasive margin, gray line, no decrease of Ber-EP4 immunoreactivity at the tumor front.

membranous Ep-CAM frequently concurs with nuclear $\beta$-catenin expression (Figure 4) makes a scenario of proteolytic Ep-CAM modifications plau-

\section{References}

1 Hase K, Shatney C, Johnson D, et al. Prognostic value of tumor 'budding' in patients with colorectal cancer. Dis Colon Rectum 1993;36:627-635. 
2 Masaki T, Muto T. Predictive value of histology at the invasive margin in the prognosis of early invasive colorectal carcinoma. J Gastroenterol 2000;35:195-200.

3 Brabletz T, Jung A, Hermann K, et al. Nuclear overexpression of the oncoprotein beta-catenin in colorectal cancer is localized predominantly at the invasion front. Pathol Res Pract 1998;194:701-704.

4 Brembeck FH, Rosario M, Birchmeier W. Balancing cell adhesion and Wnt signaling, the key role of betacatenin. Curr Opin Genet Dev 2006;16:51-59.

5 Masaki T, Goto A, Sugiyama M, et al. Possible contribution of CD44 variant 6 and nuclear betacatenin expression to the formation of budding tumor cells in patients with $\mathrm{T} 1$ colorectal carcinoma. Cancer 2001;92:2539-2546.

6 Appella E, Weber IT, Blasi F. Structure and function of epidermal growth factor-like regions in proteins. FEBS Lett 1988;231:1-4.

7 Balzar M, Bakker HA, Briaire-de-Bruijn IH, et al. Cytoplasmic tail regulates the intercellular adhesion function of the epithelial cell adhesion molecule. Mol Cell Biol 1998;18:4833-4843.

8 Tsubura A, Senzaki H, Sasaki M, et al. Immunohistochemical demonstration of breast-derived and/or carcinoma-associated glycoproteins in normal skin appendages and their tumors. J Cutan Pathol 1992;19: 73-79.

9 Cirulli V, Crisa L, Beattie GM, et al. KSA antigen EpCAM mediates cell-cell adhesion of pancreatic epithelial cells: morphoregulatory roles in pancreatic islet development. J Cell Biol 1998;140:1519-1534.

10 Kasper M, Behrens J, Schuh D, et al. Distribution of E-cadherin and Ep-CAM in the human lung during development and after injury. Histochem Cell Biol 1995;103:281-286.

11 Momburg F, Moldenhauer G, Hammerling GJ, et al. Immunohistochemical study of the expression of a $\mathrm{Mr}$ 34,000 human epithelium-specific surface glycoprotein in normal and malignant tissues. Cancer Res 1987;47:2883-2891.

12 Winter MJ, Nagtegaal ID, van Krieken JH, et al. The epithelial cell adhesion molecule (Ep-CAM) as a morphoregulatory molecule is a tool in surgical pathology. Am J Pathol 2003;163:2139-2148.

13 Salem RR, Wolf BC, Sears HF, et al. Expression of colorectal carcinoma-associated antigens in colonic polyps. J Surg Res 1993;55:249-255.

14 Packeisen J, Kaup-Franzen C, Knieriem HJ. Detection of surface antigen 17-1A in breast and colorectal cancer. Hybridoma 1999;18:37-40.

15 Basak S, Speicher D, Eck S, et al. Colorectal carcinoma invasion inhibition by CO17-1A/GA733 antigen and its murine homologue. J Natl Cancer Inst 1998;90: 691-697.

16 van Kempen LC, Rijntjes J, Claes A, et al. Type I collagen synthesis parallels the conversion of keratinocytic intraepidermal neoplasia to cutaneous squamous cell carcinoma. J Pathol 2004;204:333-339.

17 Kapiteijn E, Meershoek-Klein Kranenbarg E, Steup $\mathrm{WH}$, et al. Total mesorectal excision (TME) with or without preoperative radiotherapy in the treatment of primary rectal cancer. Prospective randomised trial with standard operative and histopathological techniques. Dutch ColoRectal Cancer Group. Eur J Surg 1999; 165:410-420.

18 Kapiteijn E, Marijnen CA, Nagtegaal ID, et al. Preoperative radiotherapy combined with total mesorectal excision for resectable rectal cancer. N Engl J Med 2001;345:638-646.

19 Nagtegaal ID, Meershoek-Klein Kranenbarg E, Hermans $\mathrm{J}$, et al. Pathology data in the central databases of multicenter randomized trials need to be based on pathology reports and controlled by trained quality managers. J Clin Oncol 2000;18:1771-1779.

20 Nagtegaal ID, Marijnen CA, Meershoek-Klein Kranenbarg E, et al. Local and distant recurrences in rectal cancer patients are predicted by the nonspecific immune response; specific immune response has only a systemic effect-a histopathological and immunohistochemical study. BMC Cancer 2001;1:7.

21 Sobin LH, Wittekind C (eds). UICC TNM Classification of Malignant Tumors, 5th edn. John Wiley \& Sons, Inc.: New York, 1997, pp 66-69.

22 Blenkinsopp WK, Stewart-Brown S, Blesovsky L, et al. Histopathology reporting in large bowel cancer. J Clin Pathol 1981;34:509-513.

23 Ueno H, Jones AM, Wilkinson $\mathrm{KH}$, et al. Histological categorisation of fibrotic cancer stroma in advanced rectal cancer. Gut 2004;53:581-586.

24 Ueno $\mathrm{H}$, Price $\mathrm{AB}$, Wilkinson $\mathrm{KH}$, et al. A new prognostic staging system for rectal cancer. Ann Surg 2004;240:832-839.

25 Balzar M, Winter MJ, de Boer CJ, et al. The biology of the 17-1A antigen (Ep-CAM). J Mol Med 1999;77: 699-712.

26 Losi L, Ponti G, Gregorio CD, et al. Prognostic significance of histological features and biological parameters in stage I (pT1 and pT2) colorectal adenocarcinoma. Pathol Res Pract 2006;202:663-670.

27 Masaki T, Matsuoka H, Sugiyama M, et al. Actual number of tumor budding as a new tool for the individualization of treatment of T1 colorectal carcinomas. J Gastroenterol Hepatol 2006;21:1115-1121.

28 Greenwald I, Rubin GM. Making a difference: the role of cell-cell interactions in establishing separate identities for equivalent cells. Cell 1992;68:271-281.

29 Hayashida Y, Honda K, Idogawa M, et al. E-cadherin regulates the association between beta-catenin and actinin-4. Cancer Res 2005;65:8836-8845.

30 Honda K, Yamada T, Hayashida Y, et al. Actinin-4 increases cell motility and promotes lymph node metastasis of colorectal cancer. Gastroenterology 2005;128:51-62.

31 Litvinov SV, Bakker HA, Gourevitch MM, et al. Evidence for a role of the epithelial glycoprotein 40 (Ep-CAM) in epithelial cell-cell adhesion. Cell Adhes Commun 1994;2:417-428.

32 Brabletz T, Jung A, Dag S, et al. Beta-Catenin regulates the expression of the matrix metalloproteinase-7 in human colorectal cancer. Am J Pathol 1999;155: 1033-1038.

33 Crawford HC, Fingleton BM, Rudolph-Owen LA, et al. The metalloproteinase matrilysin is a target of betacatenin transactivation in intestinal tumors. Oncogene 1999;18:2883-2891.

34 Takahashi M, Tsunoda T, Seiki M, et al. Identification of membrane-type matrix metalloproteinase- 1 as a target of the beta-catenin/Tcf4 complex in human colorectal cancers. Oncogene 2002;21:5861-5867.

35 Egeblad M, Werb Z. New functions for the matrix metalloproteinases in cancer progression. Nat Rev Cancer 2002;2:161-174.

36 Horkko TT, Klintrup K, Makinen JM, et al. Budding invasive margin and prognosis in colorectal cancer- 
no direct association with beta-catenin expression. Eur J Cancer 2006;42:964-971.

37 Punt CJ, Nagy A, Douillard JY, et al. Edrecolomab alone or in combination with fluorouracil and folinic acid in the adjuvant treatment of stage III colon cancer: a randomised study. Lancet 2002;360:671-677.

38 Riethmuller G, Schneider-Gadicke E, Schlimok G, et al. Randomised trial of monoclonal antibody for adjuvant therapy of resected Dukes' C colorectal carcinoma. German Cancer Aid 17-1A Study Group. Lancet 1994;343:1177-1183.

39 Sears HF, Atkinson B, Mattis J, et al. Phase-I clinical trial of monoclonal antibody in treatment of gastrointestinal tumours. Lancet 1982;1:762-765.

40 Herlyn M, Steplewski Z, Herlyn D, et al. Colorectal carcinoma-specific antigen: detection by means of monoclonal antibodies. Proc Natl Acad Sci USA 1979;76:1438-1442. 